\title{
Co-culture with human fetal epidermal keratinocytes promotes proliferation and migration of human fetal and adult dermal fibroblasts
}

\author{
ZHE WANG ${ }^{1,2}$, XIAOYU LIU ${ }^{1}$, DIANBAO ZHANG ${ }^{1}$, XILIANG \\ WANG $^{1}$, FENG ZHAO ${ }^{1}$, PING SHI $^{3}$ and XINING PANG ${ }^{1}$ \\ ${ }^{1}$ Department of Stem Cells and Regenerative Medicine, Key Laboratory of Cell Biology, \\ Ministry of Public Health and Key Laboratory of Medical Cell Biology, Ministry of Education, China Medical University, \\ Shenyang, Liaoning 110001; ${ }^{2}$ Department of Blood Transfusion, Shengjing Hospital of China Medical University, \\ Shenyang, Liaoning 110004; ${ }^{3}$ Department of General Practice, The First Affiliated Hospital of China Medical University, \\ Shenyang, Lianoning 110001, P.R. China
}

Received December 12, 2013; Accepted October 20, 2014

DOI: $10.3892 / \mathrm{mmr} .2014 .2798$

\begin{abstract}
The repair strategy for the healing of skin wounds in fetuses differs from that in adults. Proliferation and migration of dermal fibroblasts are the main mechanisms associated with skin wound healing, as well as the complex interactions between epidermal keratinocytes (KCs) and dermal fibroblasts. In order to investigate the effects of fetal skin epidermal KCs on fetal and adult human dermal fibroblasts, KCs and fibroblasts were isolated from the skin tissue of mid-gestational human fetuses and adults, and co-cultured using a Transwell ${ }^{\circledR}$ system. When fetal mid-gestational KCs were co-cultured with either fetal or adult dermal fibroblasts, the proliferative and migratory potential of the fibroblasts was significantly enhanced. Furthermore, these phenotypic changes were concomitant with the upregulation of numerous proteins including mouse double minute 2 homolog, cyclin B1, phospho-cyclin-dependent kinase 1, phospho-extracellular signal-regulated kinase, and phospho-AKT, along with C-X-C chemokine receptor 4, phospho-p38 mitogen activated protein kinase, matrix metalloproteinase (MMP)-2 and MMP-9.
\end{abstract}

Correspondence to: Professor Xining Pang, Department of Stem Cells and Regenerative Medicine, Key Laboratory of Cell Biology, Ministry of Public Health and Key Laboratory of Medical Cell Biology, Ministry of Education, China Medical University, 92 Bei'er Road, Heping District, Shenyang, Liaoning 110001, P.R. China E-mail: pangxining@126.com

Abbreviations: KCs, keratinocytes; ECM, extracellular matrix; MAPK, mitogen-activated protein kinase; MMP, matrix metalloproteinase.

Key words: human fetal epidermal KCs, human fetal dermal fibroblasts, human adult dermal fibroblasts, co-culture, proliferation, migration
Notably, no significant differences were observed between fetal and adult dermal fibroblasts in their responses to fetal mid-gestational epidermal $\mathrm{KCs}$, indicating that the cells from these two developmental stages respond in a similar manner to co-culture with KCs.

\section{Introduction}

It has been well-documented that fetuses and adults have different strategies for cutaneous wound repair. In adults, wound healing is characterized by intense inflammation and scar formation; whereas, in fetuses in the first two trimesters (at early- and mid-gestation), there is a diminished inflammatory response, decreased angiogenesis, and the absence of contraction or scar formation during the response to tissue injury (1-4). Previous research has concentrated on understanding the mechanisms underlying these different repair strategies, with the aim of providing clinical benefits for adult patients. Wound healing involves the recruitment of numerous cell types to the wound area in a temporally- and spatially-defined manner (5). Re-epithelialization largely coincides with the recruitment of dermal fibroblasts, and it is likely that the interaction between epidermal keratinocytes (KCs) and dermal fibroblasts is important during the rebuilding of tissue integrity (6). Healing of extensive wounds often results in excessive scarring, disgorging, and functional impairment of the affected area (7). This is particularly significant in the healing of large burns, and early re-epithelialization or coverage of the wounded area with autologous skin grafts limits the excessive deposition of connective tissue. The co-culture of $\mathrm{KCs}$ with fibroblasts has previously been demonstrated to stimulate paracrine loops of cytokine activation between the two cell types, which is a phenomenon that may also occur in vivo to regulate cellular function (8).

Fetal KCs are important skin cells, and numerous studies have indicated that they may be involved in fetal skin wound healing (9-11). However, the underlying mechanisms remain 
to be elucidated. Therefore, the aim of the present study was to investigate the effects of human fetal KCs, specifically the role of their secreted growth factors, on fetal and adult human dermal fibroblasts, in association with crucial parameters of tissue repair. The effects of human fetal epidermal KCs on the initial proliferative and migratory responses of dermal fibroblasts to injury were determined, as well as the signaling pathways involved.

\section{Materials and methods}

Cell culture. Fetal KCs and fibroblasts were obtained from full-thickness fetal skin specimens of miscarried fetuses (gestational age 20-23 weeks; three males and two females). Adult fibroblasts were isolated from the dermis of adult donors (aged 20-30 years; three males and two females), undergoing surgical debridement. The experiments of the present study were conducted according to the Ethical Rules for Human Experimentation, as stated in the 1975 Declaration of Helsinki, and were approved by the Ethics Committee of the Shengjing Hospital of China Medical University (Shengyang, China). Primary cultures of KCs and fibroblasts were prepared as described by previous methods (12-14). Briefly, the full-thickness skin samples were incubated at $4^{\circ} \mathrm{C}$ overnight in Dispase II (Roche Diagnostics, Indianapolis, IN, USA), and the dermal components were isolated by collagenase (1 mg/ml; Sigma-Aldrich, St Louis, MO, USA) digestion. The dermal components were then minced into $2 \mathrm{~mm}$ pieces using scissors, and the isolated fibroblasts were cultured in Dulbecco's modified Eagle's medium (DMEM; Invitrogen Life Technologies, Carlsbad, CA, USA) supplemented with $10 \%$ fetal bovine serum (FBS; Invitrogen Life Technologies). Following $0.25 \%$ trypsin digestion, the cultures of the released primary $\mathrm{KCs}$ from the fetal epidermis were initiated using tissue culture flasks coated with collagen (BD Biosciences, Franklin Lakes, NJ, USA) in Epilife ${ }^{\mathrm{TM}}$ growth medium (Life Technologies Ltd., Paisley, UK) supplemented with $1 \%$ human keratinocyte growth supplement (Life Technologies). At passage three, the KCs were co-cultured with fetal and adult fibroblasts, and used for further analysis and characterization.

Co-culture of human fetal and adult dermal fibroblasts with fetal epidermal KCs. The adult and fetal fibroblasts were co-cultured with fetal KCs in Transwell ${ }^{\circledR}$ chambers with $3 \mu \mathrm{m}$ pore filters $\left(\right.$ Costar $^{\circledR}$; Corning Incorporated, Corning, NY, USA) for 4 days. The fibroblasts were also cultured alone in DMEM supplemented with $10 \%$ FBS, this culture served as the control.

Cell proliferation assay. At days 2, 3 and 4 after the initiation of the co-culture, the proliferation of the dermal fibroblasts was measured using a tetrazolium reagent WST-1 Cell Counting kit (Beyotime Institute of Biotechnology, Haimen, China). The fibroblasts were incubated with WST-1 for $4 \mathrm{~h}$ at $37^{\circ} \mathrm{C}$. The staining intensity in the medium was determined by measuring the absorbance at a wavelength of $450 \mathrm{~nm}$, using a Safire ${ }^{2}$ microplate reader (Tecan AG, Männedorf, Switzerland), and the data were expressed as ratios of the control value. The data were compiled from three independent experiments, each performed in duplicate.
Immunofluorescence assay. Immunostaining of the $\mathrm{KCs}$ and fibroblasts was performed according to a standard protocol as previously described (12). The primary antibodies used for immunostaining were mouse monoclonal cytokeratin 14 (CK14; ab9220; 1:200) and mouse monoclonal vimentin (VIM; ab8978; 1:300) (Abcam, Cambridge, MA, USA). All of the samples were imaged using an Olympus FK-40 fluorescence microscope (Olympus Corporation, Tokyo, Japan).

Cell cycle analysis. A cell cycle analysis was conducted according to standard protocols (15). Briefly, the cells were trypsinized with $0.05 \%$ trypsin-EDTA (Sigma-Aldrich) and fixed with $70 \%$ ethanol (Sigma-Aldrich). The cells were then incubated with RNase-A (50 $\mu \mathrm{g} / \mathrm{ml}$; Sigma-Aldrich) and propidium iodide (25 $\mu \mathrm{g} / \mathrm{ml}$; Sigma-Aldrich) in phosphate-buffered saline (PBS) containing $0.1 \%$ Triton X-100 at $10^{6} / \mathrm{ml}$ (Sigma-Aldrich), for $25 \mathrm{~min}$ in the dark. Analysis of the cells in the different phases of the cell cycle was performed using flow cytometry. A minimum of 10,000 cell events per assay were acquired using a FACSCalibur ${ }^{\mathrm{TM}}$ flow cytometer (BD Biosciences).

Transwell migration assay. Fibroblast migration was measured using a Transwell ${ }^{\circledR}$ migration assay $(8 \mu \mathrm{m}$ pore size; Corning Costar), as previously described by Liu et al (14). The cells were randomly selected from eight regions per well and counted.

Western blotting. Fibroblasts were washed three times with ice-cold PBS prior to extraction of protein. Western blot analysis was performed according to standard protocols, as previously described (14). The primary antibodies used in the present study were: Rabbit polyclonal mouse double minute 2 homolog (MDM2; 1:1,000; ab58530; Abcam), rabbit polyclonal cyclin B1 (1:1,000; ab7957; Abcam), rabbit polyclonal AKT (1:1,000; \#9272; Cell Signaling Technology, Danvers, MA, USA), mouse polyclonal extracellular signal-regulated kinase (ERK; 1:1,000; \#9102; Cell Signaling Technology), mouse monoclonal matrix metalloproteinase (MMP)-2 (1:1,000; \#4022; Cell Signaling Technology), rabbit polyclonal $\mathrm{C}-\mathrm{X}-\mathrm{C}$ chemokine receptor 4 (CXCR4; ab2074; 1:1,000; Abcam), mouse polyclonal phospho-AKT (1:1,000; sc-42940; Santa Cruz Biotechnology Inc., Dallas, TX, USA), rabbit polyclonal MMP-9 (1:1,000; ab38898; Abcam), rabbit polyclonal phospho-cyclin dependent kinase (CDK)1 (1:1,000; \#9111s; Cell Signaling Technology), rabbit polyclonal CDK1 (1:1,000; sc-53219; Santa Cruz Biotechnology), rabbit polyclonal phospho-ERK (1:1,000; \#9106s; Cell Signaling Technology), mouse polyclonal phospho-p38 mitogen-activated protein kinase (MAPK; 1:1,000; sc-7973; Santa Cruz Biotechnology Inc.), and rabbit polyclonal GAPDH (1:2,000; ab37168; Abcam).

Statistical analysis. Statistical analyses were performed using SPSS version 19.0 software (SPSS Inc., Chicago, IL, USA). The data represent the mean \pm standard deviation. Comparisons between the groups were analyzed using Student's t test, and $\mathrm{P}<0.05$ was considered to indicate a statistically significant difference. The data presented in the figures are representative of three independent experiments 
A

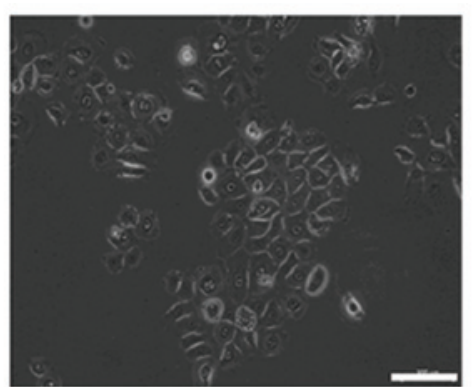

D

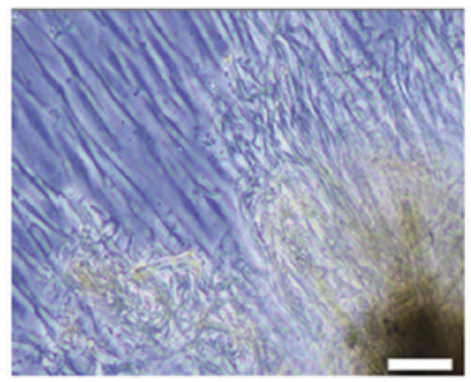

B

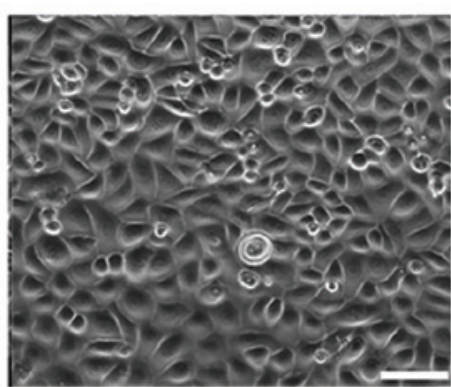

E

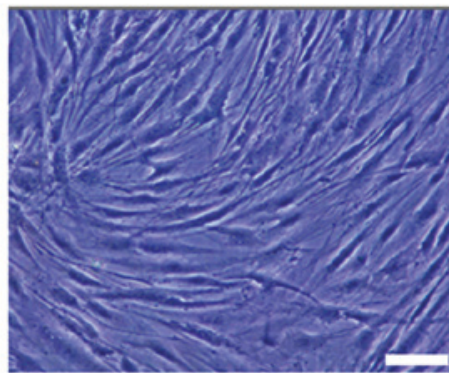

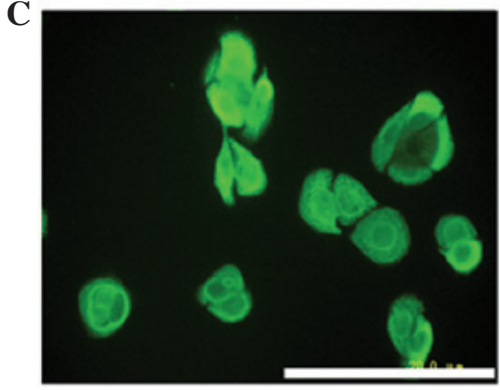

$\mathbf{F}$

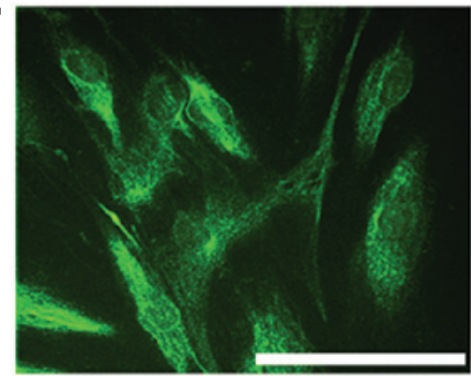

Figure 1. Identification of KCs and fibroblasts. (A and B) The morphology and growth patterns of the primary fetal KCs at days 7 and 14 after plating. (C) Cells following three passages, stained positively for cytokeratin 14 (green), a typical marker of KC cells. (D and E) The morphological appearance of primary fibroblasts on days 4 and 10 after plating. (F) Fibroblasts exhibited positive vimentin expression (green) following four passages. (A, B, D and E) Images were captured using a light microscope. (C and F) Images were captured using a fluorescence microscope. Scale bar, $200 \mu \mathrm{m}$. KC, keratinocyte.

\section{Results}

Identification of fetal epidermal KCs. The primary fetal KCs formed radiation-shaped colonies at 6-7 days post-culture (Fig. 1A). The majority of the cells acquired a typical "paving stone" shape and reached near-confluence at 14 days post-culture (Fig. 1B). In addition, specific green fluorescence was observed in the cells, indicating the expression of the typical KC surface marker CK14 (Fig. 1C).

Identification of fetal and adult dermal fibroblasts. At 3-7 days after the dermal tissue parts were plated, numerous triangular and spindle cells were shown to dissociate from the tissues (Fig. 1D). By day 10, the majority of the cells had acquired a long spindle shape and had reached near-confluence (Fig. 1E). At passage three, the fibroblasts exhibited a spindle shape and, upon reaching confluence, formed a 'whirlpool-like' pattern. Fetal and adult fibroblasts were negative for CK14, but positively expressed VIM (Fig. 1F), as determined by immunohistochemistry. These results confirm the purity of the isolated fibroblasts.

Fetal KCs promote the proliferation of fetal and adult dermal fibroblasts. To determine whether fetal epidermal KCs exhibited any biological effects on the proliferation of fibroblasts, a WST-1 analysis was conducted. Quantitative analysis showed that co-culture of fetal and adult dermal fibroblasts with fetal epidermal KCs resulted in a marked acceleration of fibroblast proliferation at days 2, 3 and 4 of culture, as compared with the control cells (Fig. 2A and B). A cell cycle analysis of the co-cultured fetal and adult dermal fibroblasts was also performed. The distribution of $S$ and $\mathrm{G}_{2} / \mathrm{M}$ phase fibroblasts was higher in the fibroblasts co-cultured with the fetal KCs, as compared with the control fibroblasts (Table I). Furthermore, western blotting was performed in order to
A

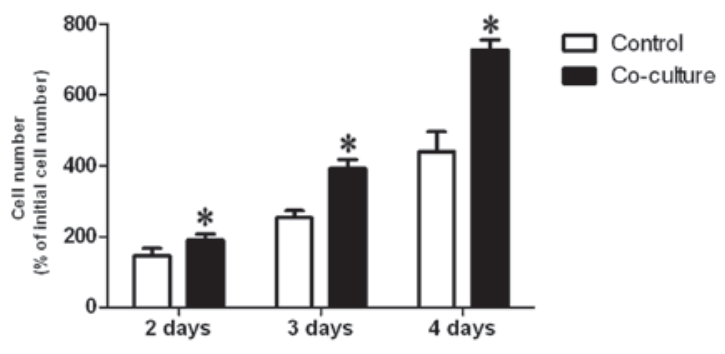

B

ADULT

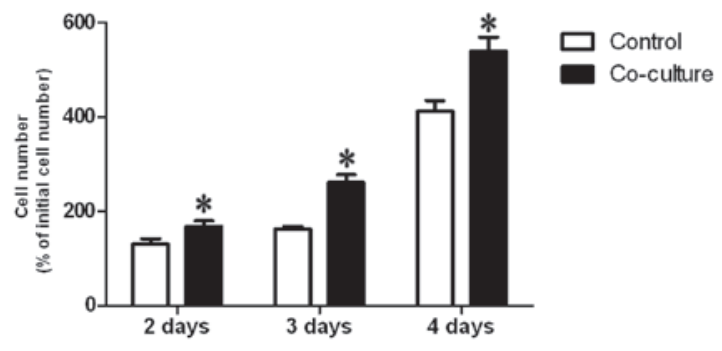

Figure 2. Stimulation of human dermal fibroblast proliferation by fetal epidermal KCs. Human (A) fetal and (B) adult dermal fibroblasts were co-cultured with fetal epidermal KCs. At 2,3 and 4 days after initiation of the co-culture, the fibroblasts were counted using a hemocytometer. Bar graphs represent the mean \pm standard deviation ( $\mathrm{n}=4$ /group). ${ }^{*} \mathrm{P}<0.05$ vs. the control group. KC, keratinocyte.

determine the expression levels of cell survival and proliferation regulators. The protein expression levels of MDM2, phospho-AKT, phospho-CDK1 and cyclin B1 were upregulated in the co-cultured fibroblasts, as compared with the control cells (Fig. 3).

Fetal KCs promote the migration of fetal and adult dermal fibroblasts. The migratory ability of fibroblasts was measured 
Table I. Cell cycle analysis of the co-culture of human skin fibroblasts with fetal KCs.

\begin{tabular}{lccc}
\hline & \multicolumn{3}{c}{$\begin{array}{c}\text { Cell cycle phase } \\
\text { distribution (\%) }\end{array}$} \\
\cline { 2 - 4 } & $\mathrm{G}_{0} / \mathrm{G}_{1}$ & $\mathrm{~S}$ & $\mathrm{G} 2 / \mathrm{M}$ \\
\hline Fetal fibroblasts & & & \\
$\quad$ Control & 63.5 & 19.2 & 17.3 \\
Co-culture with fetal KCs & 40.2 & 32.4 & 27.4 \\
Adult fibroblasts & & & \\
$\quad$ Control & 72.0 & 15.9 & 12.1 \\
Co-culture with fetal KCs & 48.7 & 25.1 & 26.2 \\
\hline
\end{tabular}

One representative experiment out of three similar experiments is presented. KC, keratinocyte.

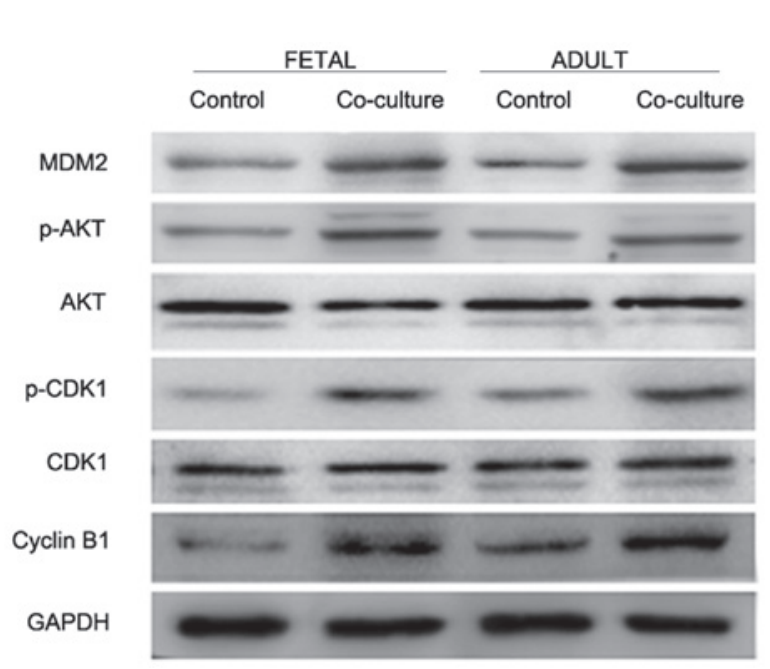

Figure 3. Protein expression levels of cell cycle regulatory molecules in human skin fibroblasts, alone or co-cultured with fetal keratinocytes, as determined by western blotting. MDM2, mouse double minute 2 homolog; CDK1, cyclin-dependent kinase 1; p-, phosphorylated-

using Transwell ${ }^{\circledR}$ assays. The fibroblasts migrated to the bottom chamber and were stained at 4 days after co-culture initiation. Quantitative analysis confirmed that the migration of the fetal and adult dermal fibroblasts to the bottom chamber was greater in the presence of fetal KCs, as compared with the control fibroblasts (Fig. 4A). Furthermore, western blotting demonstrated that fibroblasts co-cultured with fetal KCs had increased expression levels of the cell migration regulators CXCR4, phospho-ERK, and phospho38MAPK, MMP-2 and MMP-9 (Fig. 4B).

\section{Discussion}

Numerous studies have investigated the dynamics of epidermal KCs and dermal fibroblasts in co-culture, along with the influence of KCs on fibroblast proliferation and migration, two crucial steps in the wound healing process (10). However, in numerous studies, the KCs and fibroblasts used were derived from various species at different ages, and were sometimes tested in heterologous assay systems (16-20). In addition, previous research
A

FETAL

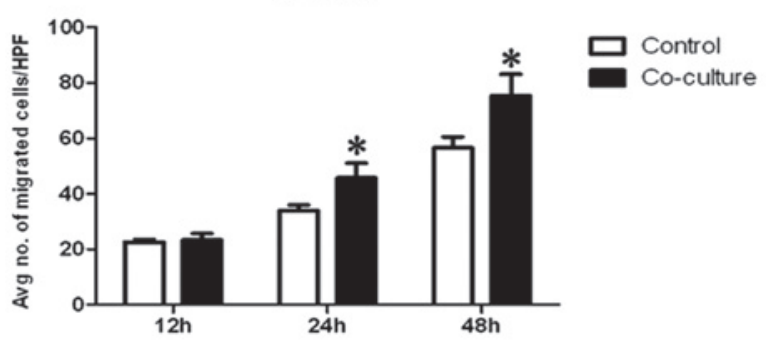

B

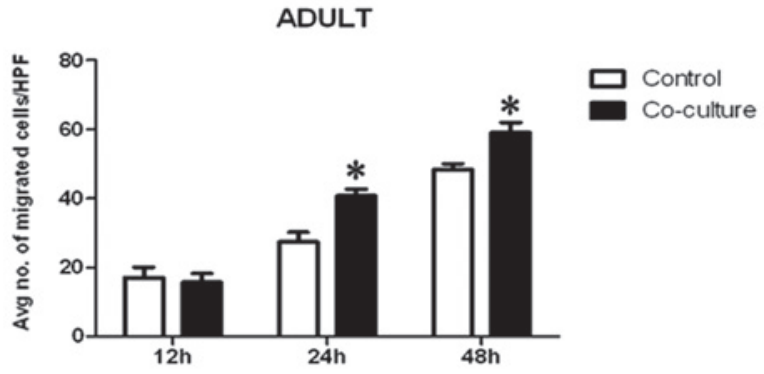

C

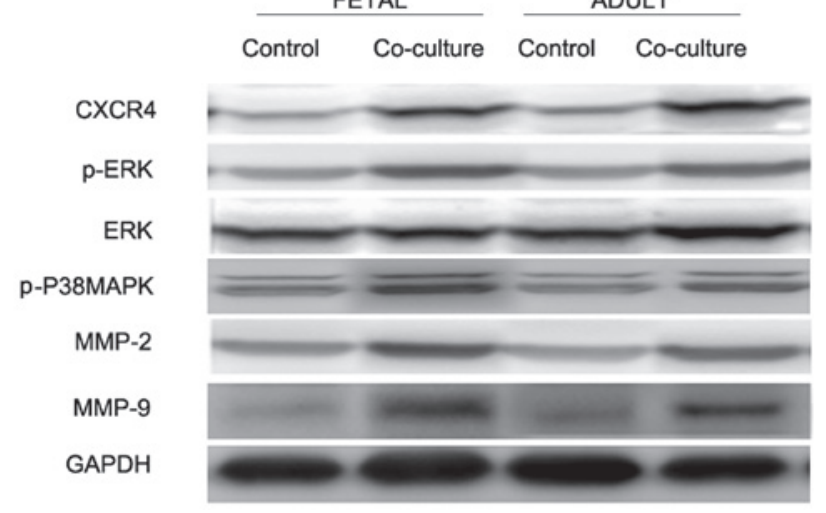

Figure 4. Analysis of the migration, and expression of cell migration and cell cycle regulators in fibroblasts alone or co-cultured with fetal keratinocytes (KCs). (A and B) Cell migratory rate of co-cultured fibroblasts, relative to control fibroblasts. Bar graphs represent the mean \pm standard deviation ( $\mathrm{n}=4$ /group). ${ }^{*} \mathrm{P}<0.05$ vs. the control group. (C) Protein expression levels of cell migration and cell cycle regulators as determined by western blotting. CXCR4, C-X-C chemokine receptor ligand; ERK; extracellular signal related kinase; MAPK, mitogen-activated protein kinase; MMP, matrix metalloproteinase; p-, phosphorylated-; HPF, high-power field.

has not always focused on fetal skin healing, but on other functions of KCs in wound healing. A previous study demonstrated that KCs from the juvenile human foreskin were not able to stimulate the proliferation and migration of dermal fibroblasts from the juvenile human foreskin (16). Conversely, human KCs were shown to stimulate the proliferation and migration of WS1 human fibroblasts in a three-dimensional extracellular matrix (ECM) (17). Furthermore, conditioned medium from cultured human KCs was shown to stimulate DNA synthesis in various human cell types $(18,19)$, and it has been shown that human KCs have a positive effect on adult skin wound healing. In a previous study it was demonstrated that KCs from newborn mice have an overall anti-fibrotic influence on fetal and postnatal fibroblasts in co-culture conditions (10). This result is in concordance with the findings of the present study.

In the present study, the co-culture of fetal and adult dermal fibroblasts with human fetal leg epidermal KCs significantly increased the proliferation of the two types of fibroblasts. 
Cutaneous wound repair is dependent on the proliferation of dermal fibroblasts, and these data suggest that mid-gestational leg KCs may stimulate fibroblast proliferation by upregulation of genes associated with the regulation of DNA synthesis. The results of the present study are concordant with previous observations of enhanced proliferation of fibroblasts (16). The protein expression levels of phospho-AKT and MDM2 in the co-cultured fibroblasts were markedly greater in the presence of fetal KCs. AKT and MDM2 are established key regulators of cell survival and proliferation. Activation of the phosphoinositide 3-kinase (PI3K)-AKT pathway has been shown to have diverse roles in fibroblasts, and the PI3K-AKT signaling pathway is known to activate a survival signal in cells, allowing them to proliferate and counteract apoptotic stimuli. Activation of the AKT signaling pathway modulates cell proliferation by transcriptional regulation of numerous genes $(21,22)$. PI3K is partly involved in facilitating cell viability by $\beta 1$ integrin interaction with the ECM, in response to mechanical forces in skin fibroblasts. Furthermore, the PI3K-AKT pathway stimulates collagen synthesis by actively promoting cell spreading and activation of the platelet-derived growth factor (23). The MDM2 protein facilitates $\mathrm{G}_{1}$-to-S phase transition by activating E2F1 and may enhance cell survival by suppressing the function of wild-type p53. Previous studies have shown that MDM2 activity is regulated by the $\mathrm{NF}-\kappa \mathrm{B}$ family of transcription factors (24-27).

To examine whether fetal KCs contributed to positive effects on the cell cycle, analysis of the cells in the different phases of the cell cycle was performed using flow cytometry. The expression levels of cell cycle regulatory components CDK1 and cyclin B1 were also evaluated. The results demonstrated that in the co-culture group, there were more fibroblasts in the $S$ and $G_{2} / M$ phases, as compared with the control group. The co-cultured fibroblasts also exhibited markedly increased expression levels of phospho-CDK1 and cyclin B1. CDK1 is known to have a critical role in cell cycle regulation, through controlling the progression of $\mathrm{G}_{1}$ to $S$, and $G_{2}$ to $M$ phase (28). CDKs and their corresponding cyclins form cyclin-CDK complexes that regulate cell cycle progression (29). Specifically, cyclin B1 is a regulatory protein involved in mitosis that forms a complex with CDK1 to form the maturation-promoting factor. Cyclin B1-CDK1 is involved in the early events of mitosis, such as chromosome condensation, nuclear envelope breakdown and spindle assembly.

The migratory ability of fibroblasts is a key factor in determining the efficiency of skin wound healing. Therefore, the present study investigated the influence of fetal $\mathrm{KCs}$ on the migratory ability of fibroblasts. One of the crucial limitations of wound healing is attributed to poor migration from adjacent healthy sites to the injured regions. Therefore, increasing the migratory ability of fibroblasts is one of the most promising approaches to improve the efficiency of skin wound healing $(30,31)$. The present study showed that co-culture with fetal KCs induced a significant increase in fibroblast cell migration. Previous studies have shown that CXCR4 and its receptor AKT, along with ERK and p38 MAPK are key mediators of fibroblast migration (32-39).

It has previously been reported that MMP-2 and MMP-9 are also regulators of fibroblast migration (40). MMPs are members of the zinc-dependent endopeptidase family and have an important role in ECM turnover. The present study demonstrated that co-culture with fetal KCs also increased the protein expression levels of MMP-2 and MMP-9.

In conclusion, the present study demonstrated that fetal $\mathrm{KCs}$ may promote the proliferation and migration of fetal and adult fibroblasts. The authors of the present study are currently investigating the effects of fetal $\mathrm{KCs}$ on other important parameters of skin wound healing, such as cell migration, contraction, and ECM accumulation and organization; and aim to determine whether keratinocyte growth factor, or other cytokines have a role in this important physiological process. The identification of the cytokine components that may contribute to complete tissue regeneration, which is a characteristic of wound healing in fetuses, is of clinical importance for treating adult wounds.

\section{Acknowledgements}

This study was supported by the National Basic Science and Development Program (973 Program grant no. 2012CB518103), the National Natural Science Foundation (grant no. 81370883), the Liaoning Province Science (grant no. 2012225080) and the Technology Plan and the Shenyang Science and Technology Program (grant no. F11-262-9-01).

\section{References}

1. Wulff BC, Yu L, Parent AE and Wilgus TA: Novel differences in the expression of inflammation-associated genes between mid- and late-gestational dermal fibroblasts. Wound Repair Regen 21: 103-112, 2013.

2. Namazi MR, Fallahzadeh MK and Schwartz RA: Strategies for prevention of scars: what can we learn from fetal skin? Int J Dermatol 50: 85-93, 2011.

3. Pouyani T, Papp S and Schaffer L: Tissue-engineered fetal dermal matrices. In Vitro Cell Dev Biol Anim 48: 493-506, 2012.

4. Cheng J, Yu H, Deng S and Shen G: MicroRNA profiling in mid- and late-gestational fetal skin: implication for scarless wound healing. Tohoku J Exp Med 221: 203-209, 2010.

5. Nowinski D, Höijer P, Engstrand T, et al: Keratinocytes inhibit expression of connective tissue growth factor in fibroblasts in vitro by an interleukin-1alpha-dependent mechanism. J Invest Dermatol 119: 449-455, 2002.

6. Fusenig NE, Limat A, Stark HJ and Breitkreutz D: Modulation of the differentiated phenotype of keratinocytes of the hair follicle and from epidermis. J Dermatol Sci 7: S142-S151, 1994.

7. Haverstock BD: Hypertrophic scars and keloids. Clin Podiatr Med Surg 18: 147-159, 2001.

8. Maas-Szabowski N, Stark HJ and Fusenig NE: Keratinocyte growth regulation in defined organotypic cultures through IL-1-induced keratinocyte growth factor expression in resting fibroblasts. J Invest Dermatol 114: 1075-1084, 2000.

9. Naik-Mathuria B, Gay AN, Yu L, et al: Fetal wound healing using a genetically modified murine model: the contribution of P-selectin. J Pediatr Surg 43: 675-682, 2008.

10. Colwell AS, Yun R, Krummel TM, Longaker MT and Lorenz HP: Keratinocytes modulate fetal and postnatal fibroblast transforming growth factor-beta and Smad expression in co-culture. Plast Reconstr Surg 119: 1440-1445, 2007.

11. Gangnuss S, Cowin AJ, Daehn IS, et al: Regulation of MAPK activation, AP-1 transcription factor expression and keratinocyte differentiation in wounded fetal skin. J Invest Dermatol 122: 791-804, 2004.

12. Hahn JM, Glaser K, McFarland KL, et al: Keloid-derived keratinocytes exhibit an abnormal gene expression profile consistent with a distinct causal role in keloid pathology. Wound Repair Regen 21: 530-544, 2013.

13. Yeh J, Green LM, Jiang TX, et al: Accelerated closure of skin wounds in mice deficient in the homeobox gene Msx2. Wound Repair Regen 17: 639-648, 2009. 
14. Liu X, Wang Z, Wang R, et al: Direct comparison of the potency of human mesenchymal stem cells derived from amnion tissue, bone marrow and adipose tissue at inducing dermal fibroblast responses to cutaneous wounds. Int J Mol Med 31: 407-415, 2013.

15. Zhang L, Aerziguli T and Guzalnur A: Establishment and characterization of a new carcinoma cell line from uterine cervix of Uyghur women. Zhonghua Bing Li Xue Za Zhi 41: 248-253, 2012 (In Chinese).

16. Wang Z, Wang Y, Farhangfar F, Zimmer M and Zhang Y: Enhanced keratinocyte proliferation and migration in co-culture with fibroblasts. PLoS One 7: e40951, 2012.

17. Laeeq S and Faust R: Modeling the cholesteatoma microenvironment: coculture of $\mathrm{HaCaT}$ keratinocytes with WS1 fibroblasts induces MMP-2 activation, invasive phenotype, and proteolysis of the extracellular matrix. Laryngoscope 117: 313-318, 2007.

18. Bernerd F: Human skin reconstructed in vitro as a model to study the keratinocyte, the fibroblast and their interactions: photodamage and repair processes. J Soc Biol 199: 313-320, 2005 (In French).

19. Kratz G, Haegerstrand A and Dalsgaard CJ: Conditioned medium from cultured human keratinocytes has growth stimulatory properties on different human cell types. J Invest Dermatol 97 1039-1043, 1991.

20. Brunet A, Bonni A, Zigmond MJ, et al: Akt promotes cell survival by phosphorylating and inhibiting a Forkhead transcription factor. Cell 96: 857-868, 1999.

21. LiN,BuX, Tian X, et al: Fatty acid synthase regulates proliferation and migration of colorectal cancer cells via HER2-PI3K/Akt signaling pathway. Nutr Cancer 64: 864-870, 2012.

22. Srivastava VK, Gara RK, Bhatt ML, Sahu DP and Mishra DP. Centchroman inhibits proliferation of head and neck cancer cells through the modulation of PI3K/mTOR pathway. Biochem Biophys Res Commun 404: 40-45, 2011.

23. Ivarsson M, McWhirter A, Borg TK and Rubin K: Type I collagen synthesis in cultured human fibroblasts: regulation by cell spreading, platelet-derived growth factor and interactions with collagen fibers. Matrix Biol 16: 409-425, 1998.

24. Lu M, Breyssens H, Salter V, et al: Restoring p53 function in human melanoma cells by inhibiting MDM2 and cyclin B1/CDK1-phosphorylated nuclear iASPP. Cancer Cell 23: 618-633, 2013.

25. Polager S and Ginsberg D: p53 and E2f: partners in life and death. Nat Rev Cancer 9: 738-748, 2009.

26. Hipfner DR and Cohen SM: Connecting proliferation and apoptosis in development and disease. Nat Rev Mol Cell Biol 5 : $805-815,2004$

27. Iwakuma $\mathrm{T}$ and Agarwal N: MDM 2 binding protein, a novel metastasis suppressor. Cancer Metastasis Rev 31: 633-640, 2012.

28. Nowack MK, Harashima H, Dissmeyer N, et al: Genetic framework of cyclin-dependent kinase function in Arabidopsis. Dev Cell 22: 1030-1040, 2012
29. Dissmeyer N, Weimer AK, Pusch S, et al: Control of cell proliferation, organ growth, and DNA damage response operate independently of dephosphorylation of the Arabidopsis Cdk1 homolog CDKA;1. Plant Cell 21: 3641-3654, 2009.

30. Makino T, Jinnin M, Muchemwa FC, et al: Basic fibroblast growth factor stimulates the proliferation of human dermal fibroblasts via the ERK1/2 and JNK pathways. Br J Dermatol 162: 717-723, 2010.

31. Liu Y, Liang C, Liu X, et al: AGEs increased migration and inflammatory responses of adventitial fibroblasts via RAGE, MAPK and NF-kappaB pathways. Atherosclerosis 208: 34-42, 2010.

32. Tarnowski M, Grymula K, Liu R, et al: Macrophage migration inhibitory factor is secreted by rhabdomyosarcoma cells, modulates tumor metastasis by binding to CXCR4 and CXCR7 receptors and inhibits recruitment of cancer-associated fibroblasts. Mol Cancer Res 8: 1328-1343, 2010.

33. Zhang D, Shao S, Shuai $\mathrm{H}$, et al: SDF-1 $\alpha$ reduces fibronectin expression in rat mesangial cells induced by TGF- $\beta 1$ and high glucose through PI3K/Akt pathway. Exp Cell Res 319: 1796-1803, 2013.

34. Blanc A, Pandey NR and Srivastava AK: Synchronous activation of ERK 1/2, p38mapk and $\mathrm{PKB} / \mathrm{Akt}$ signaling by $\mathrm{H}_{2} \mathrm{O}_{2}$ in vascular smooth muscle cells: potential involvement in vascular disease (review). Int J Mol Med 11: 229-234, 2003.

35. Song $\mathrm{J}, \mathrm{Xu} \mathrm{H}, \mathrm{Lu} \mathrm{Q}$, et al: Madecassoside suppresses migration of fibroblasts from keloids: involvement of p38 kinase and PI3K signaling pathways. Burns 38: 677-684, 2012

36. Park G, Yoon BS, Moon JH, et al: Green tea polyphenol epigallocatechin-3-gallate suppresses collagen production and proliferation in keloid fibroblasts via inhibition of the STAT3-signaling pathway. J Invest Dermatol 128: 2429-2441, 2008.

37. Li L, Zhu DL, Shen WL and Gao PJ: Increased migration of vascular adventitial fibroblasts from spontaneously hypertensive rats. Hypertens Res 29: 95-103, 2006.

38. Jeffery TK, Upton PD, Trembath RC and Morrell NW: BMP4 inhibits proliferation and promotes myocyte differentiation of lung fibroblasts via Smad1 and JNK pathways. Am J Physiol Lung Cell Mol Physiol 288: L370-L378, 2005.

39. Stawowy P, Goetze S, Margeta C, Fleck E and Graf K: LPS regulate ERK1/2-dependent signaling in cardiac fibroblasts via PKC-mediated MKP-1 induction. Biochem Biophys Res Commun 303: 74-80, 2003.

40. Yijing L, Liu $\mathrm{H}$, Yuan $\mathrm{C}$, et al: The effects of qindan-capsule-containing serum on the TGF- $\beta 1 /$ ERK signaling pathway, matrix metalloproteinase synthesis and cell function in adventitial fibroblasts. Pharm Biol 51: 712-721, 2013. 